\title{
CAIUBY LABATE Beatriz and Henrik JUNGABERLE (eds),
} The internationalization of ayahuasca

Lit Verlag, Münster, 2011

\section{David Dupuis}

\section{(2) OpenEdition}

\section{Journals}

Édition électronique

URL : https://journals.openedition.org/jsa/12931

DOI : 10.4000/jsa. 12931

ISSN : 1957-7842

Éditeur

Société des américanistes

Édition imprimée

Date de publication : 30 décembre 2013

Pagination : 226-230

ISSN : 0037-9174

\section{Référence électronique}

David Dupuis, « caluby LABAte Beatriz and Henrik Jungaberle (eds), The internationalization of ayahuasca », Journal de la Société des américanistes [En ligne], 99-2 | 2013, mis en ligne le 11 mars 2014, consulté le 03 septembre 2022. URL : http://journals.openedition.org/jsa/12931 ; DOI : https://doi.org/10.4000/ jsa.12931

Ce document a été généré automatiquement le 3 septembre 2022.

Tous droits réservés 


\section{CAIUBY LABATE Beatriz and Henrik JUNGABERLE (eds), The internationalization of ayahuasca}

Lit Verlag, Münster, 2011

David Dupuis

\section{RÉFÉRENCE}

CAIUBY LABATE Beatriz and Henrik JUNGABERLE (eds), The internationalization of ayahuasca, Lit Verlag, coll. « Performances: Intercultural studies on ritual, play and theatre »16, Münster, Allemagne, 2011, $448 \mathrm{p}$.

1 Le terme "ayahuasca »-qui signifie «liane des esprits» ou "liane des morts » en quechua - désigne à la fois une plante précise (Banisteriopsis caapi) et la préparation aqueuse dont elle est toujours l'ingrédient, unique ou principal. Cette boisson, aux puissants effets psychoactifs et émétiques, utilisée le plus souvent à des fins initiatiques, thérapeutiques ou divinatoires, constitue un outil central des chamanismes du bassin amazonien. En 1930, le Santo Daime, une religion syncrétique d'inspiration chrétienne, s'élabore autour de sa consommation et l'érige au rang de sacrement. Depuis, d'autres églises accordant au breuvage un statut central sont apparues au Brésil, comme l'União do Vegetal (UDV). À partir de la fin du $\mathrm{xx}^{\mathrm{e}}$ siècle, ces groupes religieux essaiment dans de nombreux pays, notamment en Amérique du Nord et en Europe. À la même époque, dans d'autres pays d'Amérique latine (Pérou, Colombie, Bolivie, Équateur), les usages indigènes du breuvage commencent à attirer l'attention de la population urbaine nationale ainsi que celle des Occidentaux. On voit alors apparaître un «tourisme chamanique " qui n'aura de cesse de se développer. De nombreux centres de «stages chamaniques » sont ainsi apparus, notamment en Amazonie péruvienne, près des villes de Tarapoto, Iquitos et Pucallpa. Certains ont des relais dans les pays occidentaux et fonctionnent comme des communautés thérapeutiques, proposant des stages réservés 
aux étrangers à la recherche d'«initiation». Les principales étapes de ces séjours consistent en des sessions d'ingestion de plantes vomitives en vue d'une "désintoxication ", suivies par des rituels incluant la consommation d'ayahuasca, puis de retraites impliquant celle d'autres végétaux. Ces activités sont accompagnées de conférences, d'échanges en groupe et d'entretiens avec des psychothérapeutes.

2 Le succès de ces institutions auprès des Occidentaux s'inscrit dans la continuité du mouvement New Age, qui a largement contribué à l'élaboration de la figure du " chamane », détenteur d'un savoir exotique et " primitif ", susceptible d'éclairer et de guérir l'homme occidental. L'Amazonie, région de forêt "primaire ", incarne, dans cet imaginaire, le cœur du monde primitif, un envers de l'Occident, chargé de mystères. Si l'« Indien d'Amazonie » apparaît comme susceptible de guider l'homme occidental dans sa recherche existentielle, c'est précisément parce qu'il constitue pour lui la figure d'une humanité " préservée ", épargnée par les affres de la modernité et qui aurait conservé quelque chose d'essentiel. Il constitue donc un contre-modèle à la modernité et son contact permettrait de s'en affranchir. Cette vision primitiviste invite à voir dans les rites indigènes et, particulièrement dans ceux usant de plantes hallucinogènes, des moyens pour l'homme occidental de se régénérer et de trouver un remède à des maux perçus comme inhérents à sa modernité.

3 Le développement de cette quête a conduit à l'élaboration de réseaux locaux, nationaux ou internationaux reliant des groupes indigènes, des guérisseurs métis, des leaders religieux et des " néochamanes » occidentaux. Ces différents acteurs voyagent entre les pays d'Amérique latine, mais aussi en Amérique du Nord et en Europe en vue, notamment, de conduire des rituels d'ayahuasca. Le développement de l'usage et de la visibilité de l'ayahuasca a rapidement provoqué une réaction des États nouvellement concernés par sa consommation. Des procès impliquant des consommateurs ont eu lieu dans divers pays, parallèlement à un débat sur le statut juridique de la substance. En 2005, les autorités françaises ont ainsi pris la décision de la classer au tableau des stupéfiants.

4 La rapidité et l'intensité de la globalisation de la consommation de l'ayahuasca intéressent des disciplines aussi variées que la botanique, la pharmacologie, l'anthropologie, la psychologie ou le droit. Les 27 contributions qui composent l'ouvrage The internationalization of ayahuasca tentent de dresser un état de l'art de la question. Ce livre est, pour l'essentiel, issu de la conférence organisée par l'anthropologue brésilienne Bia Labate en mai 2008 à l'université d'Heidelberg qui a rassemblé des chercheurs de ces différentes disciplines.

5 L'ouvrage débute par une préface du sociologue Charles Kaplan qui présente le rôle de l'ayahuasca dans la culture contemporaine et l'impact du phénomène de globalisation de sa consommation sur les dispositifs juridiques de contrôle de consommation des substances psychotropes. Selon lui, le cas de l'ayahuasca invite le législateur à envisager des options autres que les mesures prohibitionnistes qui étaient jusque-là employées, le DMT, principe actif de l'ayahuasca, étant inscrit au tableau 1 de la convention internationale de 1971 sur les psychotropes. Ainsi, l'ayahuasca, souvent consommée dans le cadre d'une pratique religieuse, pose la question de la frontière entre la liberté de pratique religieuse et le respect de la loi touchant la consommation et la détention des stupéfiants. Ce premier article donne le ton de l'ouvrage, qui accorde une large place aux enjeux politiques et juridiques de l'internationalisation de l'ayahuasca. 
6 L'ouvrage se compose de trois grandes parties thématiques. La première («Ayahuasca in South America and the world») dresse un tableau historique des origines de l'ayahuasca en Amérique du Sud et de sa diffusion dans le reste du monde. Nous retiendrons notamment l'article de Brabec de Mori qui défait un préjugé diffusé par de nombreux acteurs de ce champ à propos du caractère supposé « immémorial » de l'utilisation de l'ayahuasca en Amazonie. L'auteur démontre qu'il n'existe aucune preuve que l'usage de la combinaison la plus répandue (Banisteriopsis caapi et Psychotria viridis) remonte à plus de trois cents ans. Les autres articles reviennent sur l'histoire de l'ayahuasca qui, après avoir été utilisée en Amazonie, s'est diffusée dans des régions voisines comme les Andes péruviennes, sans que nous ne connaissions précisément les modalités de ce transfert. Les connaissances sont, au contraire, abondantes quant à la manière dont la substance a été adoptée par des populations métisses, puis combinée à des éléments chrétiens et africains. On apprend également comment, plus récemment, les églises brésiliennes ont ajusté leurs pratiques en vue de légitimer sa consommation dans le contexte d'un conflit juridique avec l'État brésilien. C'est ainsi qu'en 2001, l'UDV a standardisé la formule de l'ayahuasca au sein de ses églises, en vue de limiter les possibilités d'interactions chimiques dangereuses pour les participants, tandis que le Santo Daime a renoncé à l'usage de la "Santa Maria » (Cannabis sativa) qui était souvent associé à celui de l'ayahuasca.

7 La seconde section de l'ouvrage ("Medical, psychological and pharmacological issues: how safe is the use of ayahuasca?») est consacrée aux enjeux médicaux et pharmacologiques de la consommation de l'ayahuasca. On y trouve les résultats d'études sur ses risques et ses bénéfices qui semblent indiquer que la substance est peu dangereuse pour la santé physique et mentale lorsqu'elle est consommée dans le contexte d'un dispositif supervisé et que les participants ont été présélectionnés pour éviter les contre-indications médicales majeures. Deux articles donnent, par ailleurs, la voix à des " praticiens » de l'ayahuasca évoquant les bénéfices de son usage thérapeutique. Il est intéressant de noter que, dans leur effort de légitimation, les deux grands églises ayahuasqueras brésiliennes ont accepté d'accueillir des chercheurs et des spécialistes de la santé mentale dans le cadre d'études menées sur de longues périodes. L'UDV et le Santo Daime semblent même proactifs dans ce domaine en développant eux-mêmes des conditions régulant la consommation de leur sacrement, comme la présélection des participants sur des critères de santé, avec, pour but évident, d'éviter des incidents qui pourraient nuire à leur recherche de légitimation et d'institutionnalisation.

8 La troisième section de l'ouvrage ("The development of a global debate on ethics and legalization »), à nos yeux la plus intéressante, offre une synthèse inédite et détaillée des conflits juridiques touchant la consommation de l'ayahuasca dans la plupart des pays concernés (Brésil, Pays-Bas, États-Unis, Canada, France, Espagne, Italie). Pour l'essentiel, les contributions ont été rédigées par des acteurs directement impliqués dans des procès touchant l'ayahuasca en Europe et en Amérique du Nord. Nous retiendrons notamment l'article "One hundred days of ayahuasca in France " (Bourgogne) qui retrace l'histoire du processus de classification de la substance au tableau des stupéfiants français, que l'on découvre lié à deux procès impliquant une dizaine de consommateurs en France appartenant, d'une part, au Santo Daime et, d'autre part, à «La maison qui chante » (dont l'auteure est l'actuelle présidente), antenne française du centre Takiwasi (Pérou). Ce cas est ainsi exemplaire de l'internationalisation des problématiques liées à l'ayahuasca. On découvre également, dans cette partie, la description du processus par lequel le Brésil a accordé, au terme d'un long conflit juridique, la reconnaissance de l'usage de 
l'ayahuasca dans un contexte religieux. La lecture de cette section offre ainsi un riche panorama des conflits juridiques touchant la question de la consommation de l'ayahuasca dans divers pays, certains conduisant à une reconnaissance de son usage dans le cadre du respect du droit à la liberté religieuse (comme aux États-Unis en 2006), d'autres à une prohibition stricte (comme en France en 2005). Cette section a l'intérêt de montrer comment l'ayahuasca a provoqué, dans presque tous les pays concernés, une confrontation entre le régime international de classement des stupéfiants et les lois protégeant la liberté de pratique religieuse, en démontrant, dans la plupart des cas, la fragilité de ces dernières.

On regrettera toutefois l'absence d'une analyse du cas du Pérou qui, en déclarant en 2008 patrimoine culturel immatériel «les connaissances et usages traditionnels de l'ayahuasca pratiqués par les communautés natives amazoniennes» (Ugaz Villacorta 2008), a eu une approche originale de cette question. En effet, le cas péruvien ne s'appuie pas sur la liberté religieuse mais sur la reconnaissance de pratiques indigènes qui n'ont pas seulement des finalités religieuses, mais également « thérapeutiques et d'affirmation culturelle ", alors que, dans tous les autres cas (Brésil, pays anglo-saxons etc.), c'est l'usage religieux de l'ayahuasca qui est reconnu, tandis que tout usage thérapeutique est condamné. Cet exemple illustre la principale faiblesse de l'ouvrage qui se concentre trop strictement sur les religions ayahuasqueras brésiliennes, leur expansion et leurs efforts pour légitimer la consommation de leur sacrement. En effet, ces dernières ne sauraient, à elles seules, rendre compte du processus d'internationalisation de l'ayahuasca, qui implique notamment l'explosion du tourisme chamanique en Amazonie péruvienne et le développement d'un "néochamanisme » occidental. À cet égard, le recueil récemment publié par Baud et Ghasarian (2010) apporte un complément intéressant à l'ouvrage de Labate et Jungaberle. Ce dernier a toutefois le mérite de constituer une synthèse inédite, documentée et rigoureuse touchant la récente et fulgurante globalisation de la consommation de l'ayahuasca, de dresser la liste des problèmes posés par cette dernière (droit, santé publique, environnement) et de formuler des ébauches de réponse inspirantes.

UGAZ VILLACORTA Javier

2008 Résolution directoriale nationale $n^{\circ} 836$, INC, Lima.

\section{BIBLIOGRAPHIE}

BAUD Sébastien et Christian GHASARIAN (éds)

2010 Des plantes psychotropes. Initiations, thérapies et quête de soi, Imago, Paris.

\section{AUTEURS}

\section{DAVID DUPUIS}

EHESS/LAS, Paris 